\title{
A two-axis goniometer for low-temperature nuclear magnetic resonance measurements on single crystals
}

\author{
T. Shiroka, ${ }^{1,2, a)}$ F. Casola, ${ }^{1,2}$ J. Mesot, ${ }^{1,2}$ W. Bachmann, ${ }^{3}$ and H.-R. Ott ${ }^{1}$ \\ ${ }^{1}$ Laboratorium für Festkörperphysik, ETH Hönggerberg, CH-8093 Zürich, Switzerland \\ ${ }^{2}$ Paul Scherrer Institute, CH-5232 Villigen PSI, Switzerland \\ ${ }^{3}$ Departement Physik, ETH Hönggerberg, CH-8093 Zürich, Switzerland
}

(Received 4 July 2012; accepted 15 August 2012; published online 5 September 2012)

\begin{abstract}
We report on the construction of a two-axis goniometer intended for low-temperature, singlecrystal nuclear magnetic resonance (NMR) measurements. With the use of home-made and commercially available parts, our simple probe-head design achieves good sensitivity, while maintaining a high angular precision and the ability to orient samples also when cooled to liquid helium temperatures. The probe with the goniometer is adapted to be inserted into a commercial ${ }^{4} \mathrm{He}-$ flow cryostat, which fits into a wide-bore superconducting solenoid magnet. Selected examples of NMR measurements illustrate the operation of the device. () 2012 American Institute of Physics. [http://dx.doi.org/10.1063/1.4748861]
\end{abstract}

\section{INTRODUCTION}

The crystal structure of materials is often of non cubic symmetry. Consequently, their macroscopic physical properties exhibit considerable intrinsic anisotropies, such as, e.g., in electrical and thermal transport. Anisotropies are also observed in the response of single-crystalline solids to external influences, such as static magnetic fields, uniaxial stress, or directed electromagnetic radiation. These macroscopic anisotropies are mostly due to anisotropies of microscopic interactions, which depend on local configurations of the atoms occupying crystal-lattice sites. Experimentally, these interactions and their anisotropies are accessible via spectroscopic methods, such as X-ray diffraction, neutron scattering, nuclear magnetic resonance (NMR), and others.

Solid-state NMR is a useful local probe for investigating the chemical and electronic structures of selected materials, also in the presence of anisotropies. The experimental access to anisotropies, which generally accompany these structures and the related interactions, requires the possibility of varying the orientation of the single-crystalline specimen of the chosen material with respect to the axis of the RF transceiver coil and to the direction of the external magnetic field. In this way, in principle the tensors of the interaction parameters can be determined experimentally. ${ }^{1-4}$

Most of the so far developed NMR probe-head designs place the goniometer inside the radio frequency coil. ${ }^{5-8}$ This choice results in a low ratio of sample-to-coil volume (filling factor) and, consequently, in a poor signal sensitivity. For the common case of a solenoid coil, the sensitivity is proportional to the filling factor, ${ }^{9}$ defined as $\eta=V_{s} /\left(2 V_{c}\right)$, with $V_{s}$ and $V_{c}$ the sample and coil volume, respectively. ${ }^{26}$ Complementary designs, which place the goniometer outside the coil, resort to a split RF coil. ${ }^{10}$ While allowing for an easier sample rotation, this design results in a poorer coil performance compared to that of a standard solenoid. The situation is even more complicated if a second rotation axis is to be implemented.

a)Electronic mail: tshiroka@phys.ethz.ch.
Given a rotational degree of freedom in the plane perpendicular to the magnetic field direction, the availability of a second rotation axis is sufficient for covering the entire range of configurations encountered in solid-state nuclear magnetic resonance. From the practical point of view, a second rotation axis improves significantly the experimental efficiency, e.g., by avoiding interruptions of low-temperature experiments only for manually re-orienting the sample at room temperature. Despite its usefulness, the addition of a second rotation axis is generally considered as cumbersome due to constructional complexity ${ }^{11}$ and, therefore, is often not pursued.

Below we describe the development and the practical realization of a two-axis goniometer suitable for NMR measurements. Its main features include a relatively simple design, a high NMR-signal sensitivity, good precision for a chosen setting, and relatively large accessible angular ranges around both rotation axes. In addition, by choosing mostly commercially available and some in-house built parts, an easy maintenance is achieved.

\section{GONIOMETER: INSERT AND PROBE-HEAD DESIGN}

In case of angle-dependent NMR measurements, the design of the probe-head (sample holder) should also account for the possibility of cooling the sample. The operation of the goniometer at cryogenic temperatures therefore requires the probe-head to be mounted on a suitable insert. In our case, temperatures between $4 \mathrm{~K}$ and $300 \mathrm{~K}$ are achievable by means of a ${ }^{4} \mathrm{He}$ flow cryostat, which fits into the bore of a superconducting solenoid magnet (e.g., UltraShield 300, Bruker BioSpin).

The goniometer/probe-head assembly must be compact enough to fit into the magnet's bore, have flexible leads to allow for a broad range of rotations, and be made of a nonmagnetic material. Since the new probe-head was mostly meant for measuring ${ }^{63} \mathrm{Cu}$ and ${ }^{65} \mathrm{Cu}$ NMR signals, copper 


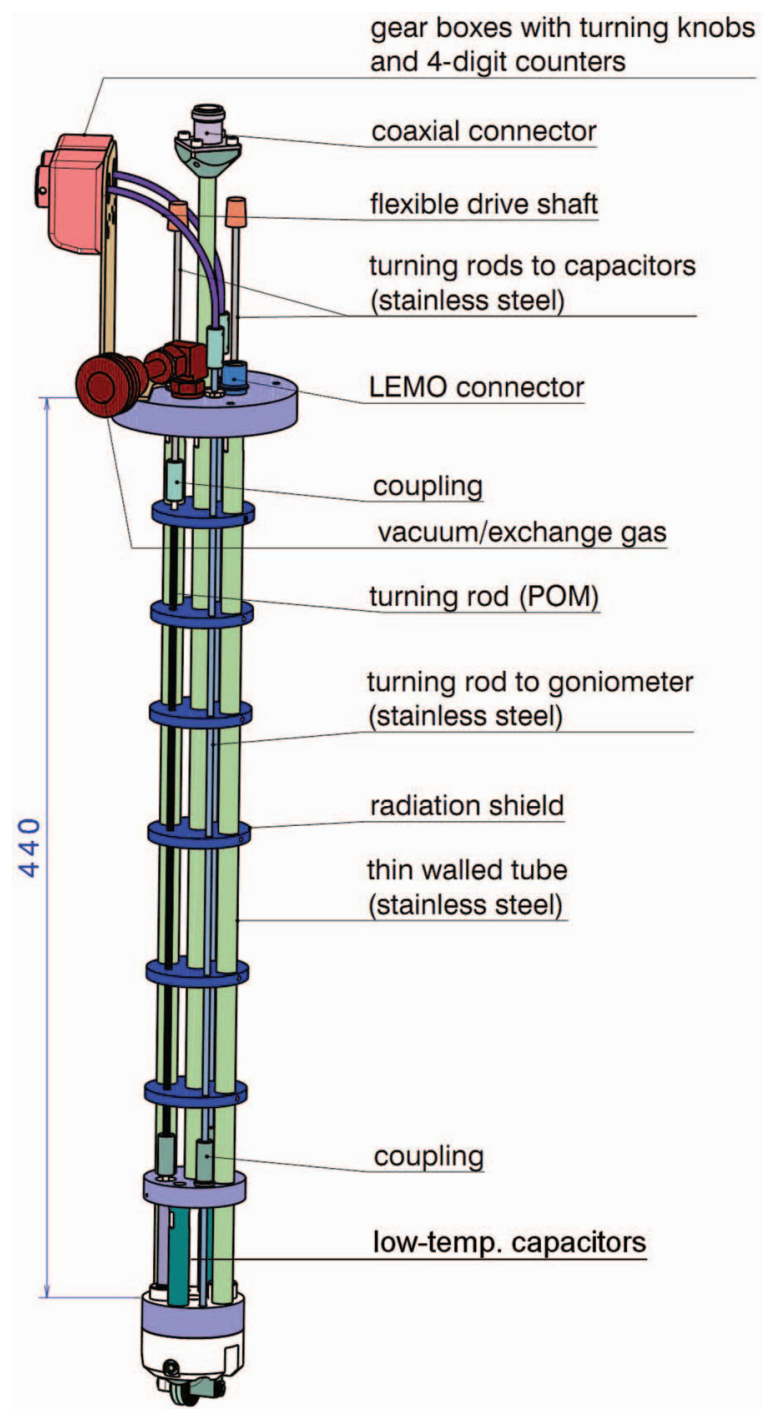

FIG. 1. Cryostat insert with the goniometer probe-head mounted at the lower end. Functions and material choices (color coded) are indicated. The shown length is in $\mathrm{mm}$.

and its alloys had to be excluded as construction materials. Aluminum (Al) was taken into account instead, since its mechanical and thermal properties represent a good compromise across the entire temperature range of interest. Indeed, with a linear thermal expansion coefficient of $23.8 \times 10^{-6} \mathrm{~K}^{-1}$ at $290 \mathrm{~K},{ }^{12}$ the expected maximum relative change of dimensions in the $4-300 \mathrm{~K}$ temperature range is smaller than $0.7 \%$. To satisfy this essential requirement, the tolerance of the rotation mechanism had to be increased at the expense of a slight reduction in angular precision. With the exception of the gears, which were commercially manufactured to our drawings, all other components were machined and assembled in the mechanical workshop of the physics department of ETH Zurich.

In Fig. 1, we show a complete 3D view of the entire goniometer device to be inserted into a continuous ${ }^{4} \mathrm{He}$ flow cryostat (CF1200, Oxford Instruments). Mounted on the top flange are a vacuum and exchange-gas connection, a vacuumtight BNC-type electrical feed-through ${ }^{13}$ for a shielded coaxial connection to the NMR coil, a vacuum-tight LEMO-type
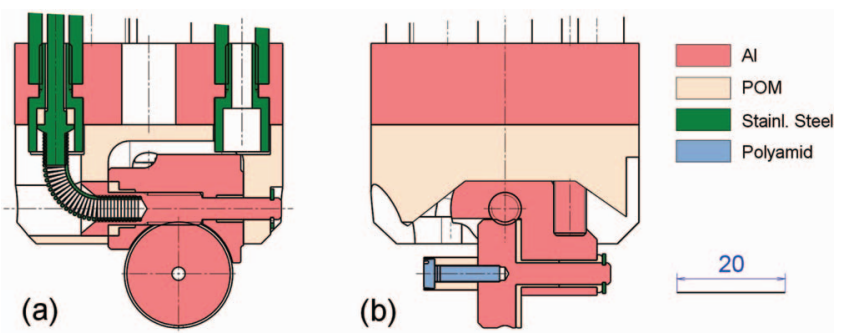

FIG. 2. Orthogonal cross sections of the goniometer head showing the constructional details. The indicated length is in $\mathrm{mm}$.

socket for the auxiliary electronic connections, and two turning rods for regulating the two capacitors of the NMR circuit mounted close to the goniometer head at the lower end of the device. Also shown are two mechanical turning devices positioned above the top flange of the insert, which provide mechanical access to regulate the orientation of the goniometer head via flexible drive shafts. The angle of orientation of each of the two revolving parts of the goniometer is indicated by a four-digit counter mounted below the knob of each gear box. The mechanical stability of the insert is given by three thin-walled stainless-steel tubes. The arrangement and the chosen materials of the turning shafts are indicated in the figure, the feed-throughs in the top flange are kept tight by O-rings.

In Fig. 2, we present two different views of the goniometer head, emphasizing important constructional details. Figures 2(a) and 2(b) are orthogonal cuts through the goniometer head, while a 3D display of the head is shown in Fig. 3. The materials from which the essential parts were machined are indicated in Fig. 2 as well. The upper part of the head is made of $\mathrm{Al}$ to which three stainless-steel tubes are fixed by stainless-steel screws. The lower part of the goniometer, serving as the holder of the two independent rotating platforms, is made of polyoxymethylene (POM). It is fixed to the upper part via two polyamide screws, not visible in the

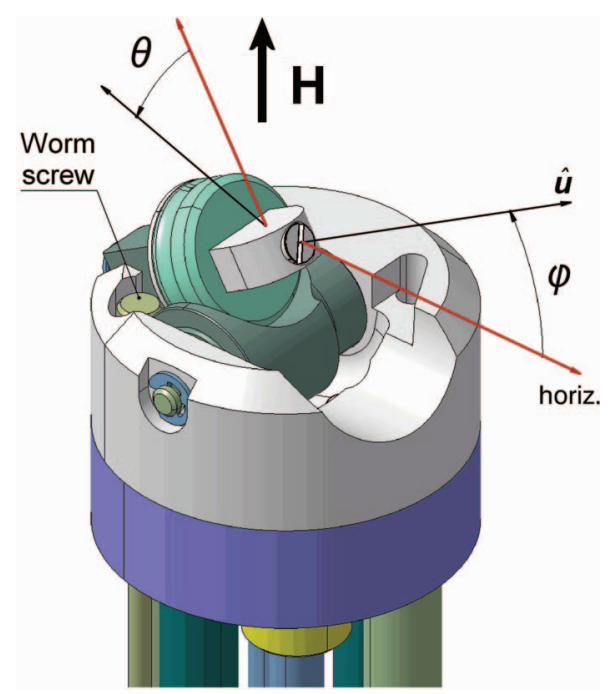

FIG. 3. Goniometer head showing the two rotation angles. The red lines indicate the reference directions for the angles $\varphi$ and $\theta$, respectively. The sample platform is fixed at the center of the $\mathrm{Al}$ wheel. 
drawings. The sample platform (Figs. 2(b) and 3), machined again from POM, is attached to an $\mathrm{Al}$ wheel and held in place by a polyamide screw. The $\mathrm{Al}$ wheel, clearly visible in Figs. 2 and 3, represents one of the rotating elements of the goniometer. It can be turned by $\pm 180^{\circ}$ via a first worm screw driven by a flexible stainless-steel drive shaft, as indicated in Fig. 2(a). The corresponding angle $\theta$ (see Fig. 3) is denoted as the proper rotation angle. The wheel is mounted on a specially machined $\mathrm{Al}$ yoke, the second rotating element, also displayed in Figs. 2 and 3. It may be turned via a second vertical drive shaft, whose rotation is transferred to the yoke via a corresponding worm screw, as indicated in Fig. 3. In this case, the accessible range of turning angles is $\pm 45^{\circ}$. Upon varying $\theta$ alone, the polar angle $(\pi / 2-\varphi)$, between the magnetic field direction $\boldsymbol{H}$ and that of the RF coil axis $\hat{\boldsymbol{u}}$, is kept constant. This is in contrast to previous two-axis designs which, in order to achieve a rotation of the sample around a chosen axis only, required the motion of one gear to be compensated by a simultaneous counter-rotation of the other. ${ }^{14,15}$

While the accessible range of the angle $\theta$ is limited only by the length of the wires connecting the movable NMR RF coil to the fixed parts, the limits of $\varphi$ are mostly imposed by the degradation of the NMR signal-to-noise $(\mathrm{S} / \mathrm{N})$ ratio occurring at large polar angles, where the axis of the detector coil approaches the orientation of the external field.

Each of the two goniometer angles may be changed manually or via a step-motor. For the first prototype, manual operation, as shown in Fig. 1 and described above, was chosen. Repeated tests have shown a good reproducibility (to within $0.5^{\circ}$ ) of the sample's angular position. Elastic hysteresis issues, common to goniometer heads, are minimized in our case through the use of mechanical components working under low-load conditions. All parts of the goniometer were chosen to be suitable for operation under vacuum conditions and in a cryogenic environment. As mentioned above, in order to ensure the mobility of the different parts also at low temperatures, special material combinations with favorable thermalexpansion coefficients and low friction were implemented. In particular, since we use the polycrystalline form of POM (Delrin), it was necessary to account for the average linear thermal expansion coefficient $1 / 3\left(2 \alpha_{\perp}+\alpha_{\|}\right)$, calculated from the published values of $\alpha_{\|}$and $\alpha_{\perp},{ }^{16}$ the linear expansion coefficients parallel and perpendicular to the organic chains, respectively. Finally, the surface of all parts susceptible to friction, namely all threadings involved in the rotational motions covering the angles $\varphi$ and $\theta$, were hardened by commercial electrochemical processes. In order to reduce friction, all the rotation axes are supported by POM bearings positioned between the axes and the bearing cases.

To achieve a high NMR sensitivity, a solenoidal coil tightly wound around the sample, providing a high filling factor, was used. Typically, the coil with the sample is mounted on the flat face of the sample platform so that the coil axis coincides with the $\hat{\boldsymbol{u}}$-axis of the goniometer (see Fig. 3). On several occasions, we found it convenient to add another coil, wound on top of the first, whose axis is orthogonally oriented with respect to both the axis of the first coil and to the sampleplatform surface. The second coil served to avoid the gradual loss of sensitivity occurring when the angle between the axis of the original coil and the magnetic field direction exceeds $45^{\circ}$, without having to unmount the sample. Changing the electrical contacts from the first to the second coil, of course, required lifting the insert out of the cryostat. Under most unfavorable conditions, with respect to the mutual orientation between field and one of the RF coil axes, the NMR sensitivity would drop at most by a factor of $\sqrt{2}$ with respect to its maximum value. At this point, no attempts were made to extend and optimize the performance of the RF circuit to frequencies above $200 \mathrm{MHz}$.

\section{APPLICATIONS AND DISCUSSION}

In this section, we show the results of a few angledependent, low-temperature NMR measurements, carried out using the new goniometer.

Our first example concerns $\mathrm{BaCu}_{2} \mathrm{Si}_{2} \mathrm{O}_{7}$, a model spinchain compound where we intended to study the system's dimensional crossover upon temperature changes via NMR measurements. ${ }^{17}$ The ${ }^{29} \mathrm{Si}$ NMR resonances of $\mathrm{BaCu}_{2} \mathrm{Si}_{2} \mathrm{O}_{7}$ depend strongly on sample orientation, especially in its magnetically ordered phase. Here, even small misalignments entail significant changes of the NMR line-shapes and positions. The use of the goniometer head offered the possibility to perform in situ sample orientation, ${ }^{18,19}$ a necessary first step for subsequent studies. From the patterns shown in Fig. 4, we could unambiguously identify the crystalline $a$ and $b$ axes, and then proceed with our study, where the externally applied field had to be kept in the crystal's $a b$ plane.

As a second example, we present the orientation dependence of ${ }^{31} \mathrm{P}$ NMR patterns obtained by probing a single crystal of $\mathrm{BiCu}_{2} \mathrm{PO}_{6}$. Previous studies have shown that this compound hosts zigzag shaped two-leg spin ladders. ${ }^{20}$ The ladders, formed by $S=\frac{1}{2} \mathrm{Cu}$ ions, run along the $b$ direction of the crystal lattice, while the $I=1 / 2{ }^{31} \mathrm{P}$ nuclei act as local probes of the magnetism and spin dynamics. Details about the physical properties of the spin system of this compound are presented and discussed in Ref. 21. The sample was initially mounted so that the $c$ axis of the orthorhombic crystal lattice coincided with the goniometer axis $\hat{\boldsymbol{u}}$ (i.e., $\theta=\varphi=0$ ), while

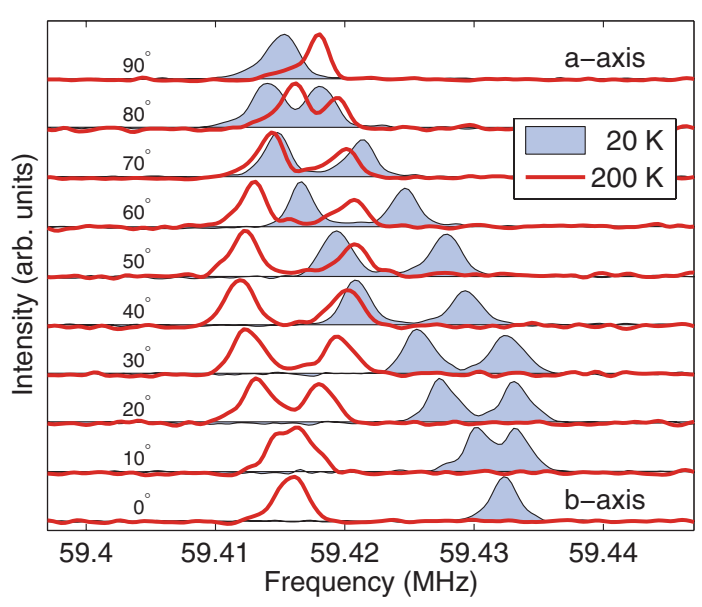

FIG. 4. Single-crystal ${ }^{29} \mathrm{Si} \mathrm{NMR} \mathrm{spectra} \mathrm{of} \mathrm{BaCu}_{2} \mathrm{Si}_{2} \mathrm{O}_{7}$ recorded at $20 \mathrm{~K}$ (shaded areas) and $200 \mathrm{~K}$ (thick red lines) in $10^{\circ}$ increments for a rotation about the crystalline $c$ axis. 

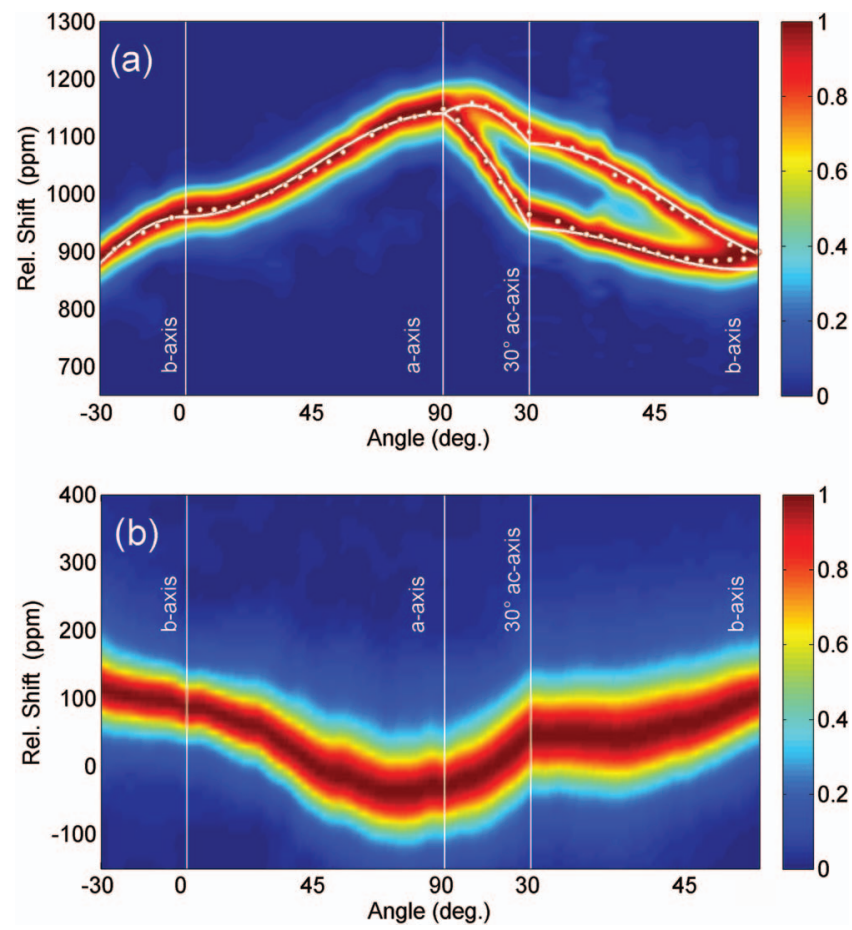

FIG. 5. Single-crystal ${ }^{31} \mathrm{P}$ spectra of $\mathrm{BiCu}_{2} \mathrm{PO}_{6}$ recorded at $290 \mathrm{~K}$ (a) and at $5 \mathrm{~K}$ (b) in $10^{\circ}$ increments for rotations about different crystalline axes. Colors reflect the normalized signal intensity, whereas dots show peak maxima. Vertical lines indicate angular positions where selected crystalline axes coincide with the applied magnetic field direction. The continuous lines in the top panel show numerical fits using analytic expressions for the NMR shift assuming a fully field-polarized electronic spin system.

the $b$ axis was parallel to the applied magnetic field $\boldsymbol{H}$. By using the two available degrees of freedom, we could monitor the shapes and strengths of the NMR signals at different crystal orientations with respect to the direction of the applied magnetic field.

Figure 5 shows the rotation patterns of the renormalized signal height obtained at both high and low temperatures. The availability of more than one rotation axis is a clear advantage, since in this case all the relevant tensorial components can be evaluated from a single rotation pattern of the undoped (Fig. 5) and 1\% Zn-doped compound (not shown), where line degeneracies due to crystal symmetry are lifted. This is best illustrated in the top panel of Fig. 5, where the theoretical curve, using the experimentally extracted parameters, represents an excellent fit to the measured pattern. The differences in the angular dependence of the two reported patterns are due to low-temperature magnetic anisotropies, as described in detail in a forthcoming paper.

Future developments of this two-axis goniometer will include the use of stepper motors and angular position sensors for the automatic setting and measuring of the sample alignment. Optical encoders or Hall sensors represent two possible choices for the angle sensors, ${ }^{22,23}$ already in use in other
NMR setups reported in the literature. ${ }^{24,25}$ In the latter case, since the induced voltage depends on the projection of the magnetic flux density, the true angular position of the goniometer can be established by employing two different Hall probes, rotated by $90^{\circ}$ with respect to one another. ${ }^{25}$ An updated version of our goniometer, with automated rotations of the sample using step motors for fully computer-controlled measurements, is in the planning stage.

\section{ACKNOWLEDGMENTS}

We would like to thank Professor T. Vosegaard and Dr. B. Graneli for helpful preliminary discussions and Mr. A. Stuker for technical assistance during the construction of the goniometer head. This work was financially supported in part by the Schweizerische Nationalfonds zur Förderung der Wissenschaftlichen Forschung (SNF) and the NCCR research pool MaNEP of SNF.

${ }^{1}$ L. J. Mueller, Concepts Magn. Reson. 38, 221 (2011).

${ }^{2}$ M.-A. Vachon, G. Koutroulakis, V. F. Mitrović, A. P. Reyes, P. Kuhns, R. Coldea, and Z. Tylczynski, J. Phys.: Condens. Matter 20, 295225 (2008).

${ }^{3}$ A. Kiss and Y. Kuramoto, J. Phys. Soc. Jpn. 77, 72 (2008).

${ }^{4}$ C. P. Poole, Jr., and H. A. Farrach, Theory of Magnetic Resonance, 2nd ed. (Wiley, New York, 1987), Chap. 6.

${ }^{5}$ A. Pines, J. J. Chang, and R. G. Grifn, J. Chem. Phys. 61, 1021 (1973).

${ }^{6}$ S. Pausak, J. Tegenfeldt, and J. S. Waugh, J. Chem. Phys. 61, 1338 (1974).

${ }^{7}$ W. S. Veeman, Philos. Trans. R. Soc. London, Ser. A 299, 629 (1981).

${ }^{8}$ T. Vosegaard, V. Langer, P. Daugaard, E. Hald, H. Bildsøe, and H. J. Jakobsen, Rev. Sci. Instrum. 67, 2130 (1996).

${ }^{9}$ D. I. Hoult and R. E. Richards, J. Magn. Reson. 24, 71 (1976).

${ }^{10}$ H. Hauser, C. Radloff, R. R. Ernst, S. Sundell, and I. Pascher, J. Am. Chem. Soc. 110, 1054 (1988)

${ }^{11}$ T. Vosegaard, E. Hald, P. Daugaard, and H. J. Jakobsen, Rev. Sci. Instrum. 70, 1771 (1999)

${ }^{12}$ H. M. Otte, W. G. Montague, and D. O. Welch, J. Appl. Phys. 34, 3149 (1963).

${ }^{13}$ D. Griffiths and I. Coole, J. Phys. E: J. Sci. Instrum. 6, 524 (1973).

${ }^{14}$ R. Herzog, J. E. Evetts, R. E. Somekh, and P. A. Pullan, in Proceedings of the 7th International Workshop on Critical Currents in Superconductors, edited by H.Webber (World Scientific, Singapore, 1994), pp. 391.

${ }^{15}$ R. Herzog and J. E. Evetts, Rev. Sci. Instrum. 65, 3574 (1994).

${ }^{16}$ G. K. White, T. F. Smith, and J. A. Birch, J. Chem. Phys. 65, 554 (1976).

${ }^{17} \mathrm{~F}$. Casola et al., "Dimensional crossover of spin chains in a transverse staggered field: an NMR study," Phys. Rev. B (submitted).

${ }^{18}$ J. B. Milstein, Rev. Sci. Instrum. 61, 2581 (1990).

${ }^{19}$ T. Vosegaard, E. Hald, V. Langer, H. J. Skov, P. Daugaard, H. Bildsøe, and H. J. Jakobsen, J. Magn. Reson. 135, 126 (1998).

${ }^{20}$ F. Abraham, M. Ketatni, G. Mairesse, and B. Mernari, Eur. J. Solid State Inorg. Chem. 31, 313 (1994).

${ }^{21}$ F. Casola, T. Shiroka, S. Wang, K. Conder, E. Pomjakushina, J. Mesot, and H.-R. Ott, Phys. Rev. Lett. 105, 067203 (2010).

${ }^{22}$ W. Kester, in Sensor Technology Handbook, edited by J. S. Wilson (Newnes, Burlington, MA, 2005), pp. 379-400.

${ }^{23}$ J. Fraden, Handbook of Modern Sensors: Physics, Designs, and Applications (Springer, New York, 2010).

${ }^{24}$ S. Mamone, A. Dorsch, O. G. Johannessen, M. V. Naik, P. K. Madhu, and M. H. Levitt, J. Magn. Reson. 190, 135 (2008).

${ }^{25}$ A. Straub, B. Sc. thesis, Brown University, Providence, RI, 2006.

${ }^{26}$ The factor 2 reflects the fact that in a solenoid coil only half of the $B_{1}$ field is contained within the coil. 